\title{
YWHAE/FAM22 Fusion Gene
}

National Cancer Institute

\section{Source}

National Cancer Institute. YWHAE/FAM22 Fusion Gene. NCI Thesaurus. Code C127057.

A fusion gene that results from a chromosomal translocation $t(10 ; 17)(q 22 ; p 13)$ which fuses the exon 5 the YWHAE gene with exon 2 of the FAM22A (NUT M2A), FAM22B (NUT M2B), or FAM22E (NUTM2E) gene. These fusions are associated with high grade endometrial stromal sarcomas and clear cell sarcomas of the kidney. 